\title{
Ocorrência e distribuição espacial e temporal do coleóptero Naupactus curtus em cafeeiros de Minas Gerais, Brasil
}

\author{
Occurrence, spatial and temporal distribution of the coleopteran Naupactus curtus on coffee \\ plants from Minas Gerais, Brazil
}

\author{
Flávio Lemes Fernandes ${ }^{\mathrm{I}}$ Marcelo Coutinho Picanço ${ }^{\mathrm{II}}$ Rodrigo Soares Ramos ${ }^{\mathrm{II}}$ \\ Jorgiane da Silva Benevenute ${ }^{\mathrm{II}}$ Maria Elisa de Sena Fernandes ${ }^{\mathrm{III}}$
}

\begin{abstract}
Não há registro de Naupactus curtus em Minas Gerais, e as poucas ocorrências em outros Estados brasileiros são escassas de informações acerca da sua distribuição espacial em cafeeiros. Assim, este trabalho tem por objetivos relatar a ocorrência dessa praga em Minas Gerais e estudar a sua distribuição espacial e temporal em quatro lavouras de Coffea arabica, em Ponte Nova e Jaboticatubas. Os insetos foram monitorados mensalmente. $N$. curtus foi encontrado nessas duas áreas e apresentou maiores densidades nas bordas das lavouras.
\end{abstract}

Palavras-chave: Coffea arabica, dispersão, densidade populacional.

\section{ABSTRACT}

There were no reports of Naupactus curtus in Minas Gerais, and the few occurrences in other Brazilian States bring is little of information about its spatial distribution on coffee plants. This work aimed to report the occurrence of this potential in Minas Gerais and study its their spatial and temporal distribution dynamics on crops of Coffea arabica. The study was conducted in coffee plants in Ponte Nova and Jaboticatubas, with the evaluation of density being monthly. N. curtus was found in two areas in Minas Gerais, presenting higher densities at the edges of the crops.

Key words: Coffea arabica, dispersion, populational density.
Recentes pesquisas têm registrado a ocorrência de Naupactus curtus (Coleoptera: Curculionidae: Entiminae: Naupactini) em São Paulo, na Bahia, no Paraná e em Santa Catarina, alimentandose de folhas de café (Coffea arabica L.), acerola (Malpighia glabra L.), amora (Morus alba L.) e Citrus sinensis (L.) Osbeck. As larvas de Naupactus são brocadoras de raízes, causando sérios danos ao sistema radicular das plantas e, em Citrus, sabe-se que reduzem o desenvolvimento e a produção (LANTERI et al., 2002). Além disso, as lesões nas raízes facilitam a ocorrência de doenças, como o fungo Phytophthora spp., agente causal da gomose, que pode levar a planta à morte (GUEDES et al., 2002). $\mathrm{O}$ adulto de $N$. curtus possui de 10 a $12 \mathrm{~mm}$ de comprimento, corpo coberto com escamas marrons, uma listra branca ao longo da sutura elitral e um par de listras brancas oblíquas nas laterais dos élitros, depositam seus ovos nas plantas ou no solo e, por serem o estágio alado, são os responsáveis pela disseminação da praga nas lavouras (SÁNCHEZ-SOTO et al., 2005; LANTERI et al., 2002). Dessa forma, estudos sobre a época de ocorrência e o padrão espacial dos adultos são importantes para criar estratégias de controle.

Alguns insetos anteriormente não considerados pragas e restritos a poucas áreas no

IDepartamento de Ciências Agrárias, Universidade Federal de Viçosa (UFV), Campus Rio Paranaíba, 38810-000, Rio Paranaíba,

MG, Brasil. E-mail: flaviofernandes@ufv.br. Autor para correspondência.

IIDepartamento de Biologia Animal, UFV, Viçosa, MG, Brasil.

IIIDepartamento de Biologia Geral, UFV, Viçosa, MG, Brasil. 
Brasil são hoje importantes pragas, causando prejuízos consideráveis para a agricultura do país. Scaptocoris castanea Perty (Hemiptera: Cydnidae), por exemplo, era de ocorrência esporádica nas culturas de soja (Glycine Max L.) e, a partir de 1984, tornou-se praga importante em lavouras de soja, algodão, milho-safrinha e arroz, principalmente em Mato Grosso, São Paulo e Minas Gerais (NAKANO et al., 2001; OLIVEIRA et al., 2003). O fato de os insetos passarem de uma ocorrência restrita a uma ampla distribuição ao longo do tempo pode estar associado ao seu poder de dispersão. Assim, o presente trabalho tem como objetivos relatar a ocorrência de $\boldsymbol{N}$. curtus em Minas Gerais, onde a espécie ainda não havia sido registrada, e estudar a sua distribuição espacial e temporal em quatro lavouras cafeeiras.

O estudo foi realizado em Minas Gerais, em quatro lavouras (talhões) de $\boldsymbol{C}$. arabica (var. catuaí vermelho), linhagem IAC 144, sendo três lavouras (talhões 1, 2 e 3, com 15, 12 e 10ha, respectivamente) localizadas na Zona da Mata, Ponte Nova ( $21^{\circ} 20^{\prime}$ sul e $43^{\circ} 13^{\prime}$ oeste; altitude: $\left.430 \mathrm{~m}\right)$, e a quarta lavoura, de 20ha, localizada em Jaboticatubas ( $19^{\circ} 30^{\prime}$ sul e $43^{\circ} 44^{\prime}$ oeste; altitude: $1858 \mathrm{~m})$, região central do Estado. As vistorias ocorreram de setembro de 2006 a dezembro de 2007 em Jaboticatubas, e de setembro de 2007 a março de 2009 nas lavouras em Ponte Nova. Dados climáticos de velocidade do vento, precipitação pluviométrica e temperatura foram obtidos nas duas regiões. Em Jaboticatubas, os meses mais e menos quentes foram, respectivamente, novembro (temperatura média de $25^{\circ} \mathrm{C}$ ) e julho (temperatura média de $17^{\circ} \mathrm{C}$ ). Os meses mais chuvosos foram janeiro e fevereiro (média de $27 \mathrm{~mm}$ mensal). O mês com maior velocidade do vento foi agosto $\left(10 \mathrm{~m} \mathrm{~s}^{-1}\right)$. Em Viçosa, os meses mais e menos quentes também foram, respectivamente, janeiro (temperatura média de $31^{\circ} \mathrm{C}$ ) e julho (temperatura média de $10^{\circ} \mathrm{C}$ ). Os meses mais chuvosos foram novembro e dezembro (média de 59mm mensal) e precipitação média mensal de $35 \mathrm{~mm}$. O mês com maior velocidade do vento foi fevereiro $\left(6 \mathrm{~m} \mathrm{~s}^{-1}\right)$. Entre os elementos climáticos, temperatura, precipitação pluviométrica e velocidade do vento são os principais fatores relacionados à dinâmica populacional de insetos em agroecossistemas (WALLNER, 1987).

As avaliações das densidades de adultos de $N$. curtus nos cafeeiros foram realizadas por meio da técnica de amostragem batida de bandeja em dois ramos, na região mediana de 120 plantas, da extremidade para o meio do ramo, em um total de 240 batidas de bandeja por lavoura. Os adultos presentes na bandeja foram coletados para compor uma coleção de referência na
Coleção Entomológica da Universidade Federal de Viçosa. Calculou-se a densidade média de adultos por meio do número de besouros/100 plantas/lavoura. Além disso, em cada par de folhas do quarto entrenó, foi contado o número de folhas com lesões de adultos de $N$. curtus em cada talhão. As amostragens foram feitas em indivíduos de plantas distanciados $30 \mathrm{~m}$, no sentido das fileiras do cafeeiro, em 120 plantas lavoura ${ }^{-1}$. Para verificar se os danos dos insetos às plantas nas áreas avaliadas foram representativos, foi feita correlação de Pearson $(\mathrm{P} \leq 0,05)$ do número de adultos/bandeja com a proporção de folhas lesionadas em todas as lavouras e meses de avaliação. Testou-se a dependência espacial pelo índice de Moran (I) (a P $\leq 0,05)$, pelo teste Z. O (I) testa se o inseto se distribui de forma irregular, uniforme ou mais agregado, e valores de I próximos a 1 indicam alta dependência espacial, enquanto valores próximos a zero indicam baixa ou ausência de dependência espacial.

Naupactus curtus foi encontrado nas duas áreas estudadas em Minas Gerais. Em Jaboticatubas, a espécie foi registrada a partir de abril de 2007, aumentando a sua densidade até atingir o pico em agosto, decrescendo consideravelmente em setembro e desaparecendo a partir de outubro (Figura1 I). Observou-se correlação positiva da precipitação pluviométrica $(\mathrm{r}=0,64 ; \mathrm{P} \leq 0,05)$ e da velocidade do vento $(\mathrm{r}=0,71 ; \mathrm{P} \leq 0,05)$ com a densidade média de $\mathrm{N}$. curtus na lavoura dessa região. Já em Ponte Nova os adultos ocorreram de abril a dezembro de 2008, com os picos de densidade variando entre as lavouras, sendo as maiores densidades populacionais registradas entre os meses de outubro e novembro de 2008 (Figura 1 I). Nesse local, a correlação da densidade de adultos com a velocidade do vento foi positiva $(\mathrm{r}=0,61 ; \mathrm{P} \leq 0,05)$, porém não significativa ( $\mathrm{r}=0,26 ; \mathrm{P}>0,05)$ com a precipitação pluviométrica. Não se observou correlação da temperatura com as densidades de $N$. curtus.

A correlação positiva entre adultos e a precipitação pluviométrica pode ser explicada em razão da umidade que favorece as larvas no solo, uma vez que essas condições beneficiam a fauna do solo (CAMPANHOLA, 2002), não influenciando o adulto. A correlação negativa tem sido verificada entre insetos adultos e precipitação (BACCA et al., 2006). Verificouse uma correlação positiva do número de adultos por amostra com a proporção de folhas lesionadas ( $\mathrm{r}=0,76$; $\mathrm{P} \leq 0,05)$. Esse fato indica que o aumento dos danos desse inseto em café representa maior número de adultos/batida de bandeja. Assim, futuramente, esse método de amostragem pode vir a ser usado no monitoramento dessa praga. O besouro estudado apresenta restrita e baixa ocorrência nos cafeeiros 


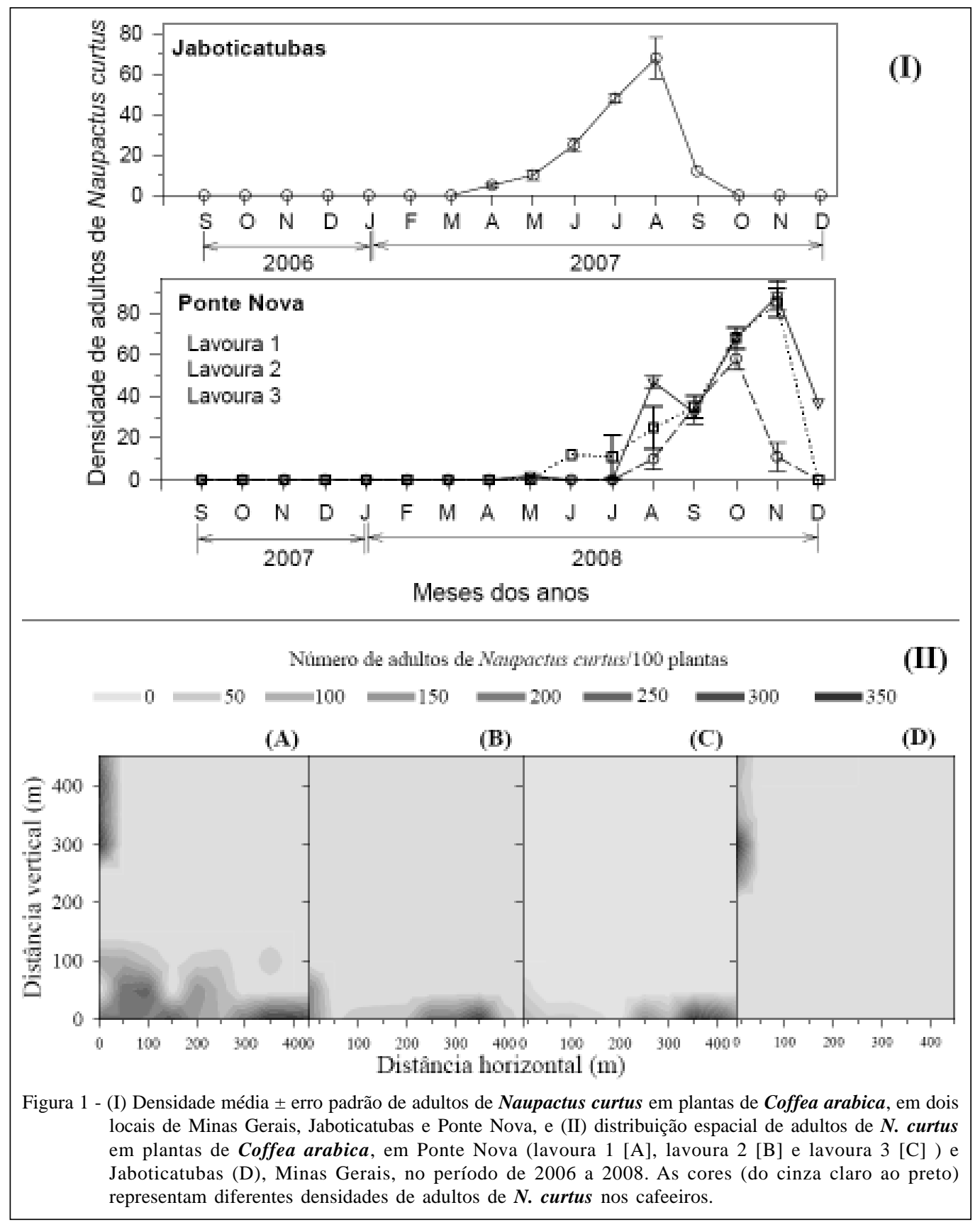

brasileiros (SÁNCHEZ-SOTO et al., 2005), sendo necessários mais estudos em outras regiões do Brasil para avaliar o seu potencial de dispersão. Estudos sobre outros fatores, como característica da planta hospedeira e inimigos naturais, deveriam ser abordados em próximos trabalhos (WILLIAMS et al., 2008). Dessa forma, trabalhos futuros devem ser direcionados aos fatores que determinam o ataque de $N$. curtus nessas regiões.
Observou-se dependência espacial positiva da densidade dos adultos de $\boldsymbol{N}$. curtus tanto nas três lavouras de Ponte Nova ( $\mathrm{I}=0,57 ; \mathrm{Z}=3,42, \mathrm{P} \leq 0,05 ; \mathrm{I}=0,68$; $\mathrm{Z}=4,11, \mathrm{P} \leq 0,05 \mathrm{e} \mathrm{I}=0,77 ; \mathrm{Z}=5,23, \mathrm{P} \leq 0,05$, nas lavouras 1 , 2 e 3 , respectivamente), quanto nas lavouras em Jaboticatubas ( $I=0,56 ; Z=3,42, P \leq 0,05)$. Essa dependência espacial é evidenciada pela distribuição agregada de adultos de $\boldsymbol{N}$. curtus (Figura 1 II). Além disso, observou-se tendência de início do ataque pelas 
laterais da região avaliada nas lavouras, onde a densidade de adultos é marcadamente maior (Figura 1 II). A maior densidade de insetos nas bordas das lavouras pode indicar que as infestações iniciam nesses locais. Esse fato pode ser verdadeiro em razão da correlação positiva entre o número de adultos e a velocidade do vento, uma vez que a dispersão de insetos-praga muitas vezes ocorre por meio dos ventos (STINNER, 1983). BUSOLI et al. (1994) relatam que a densidade dos adultos de Anthonomus grandis (Coleoptera: Curculionidae) tendeu a ser mais concentrada nas bordas da cultura do algodoeiro (focos iniciais do bicudo).

O presente estudo registrou pela primeira vez a ocorrência de $\boldsymbol{N}$. curtus em Minas Gerais, em duas regiões, sendo uma na Zona da Mata e a outra na região central, relatando as épocas e os locais de maior incidência desse inseto e trazendo informações importantes para o monitoramento e o efeito do clima sobre a densidade. Além disso, as informações sobre a presença desse inseto nas bordas das lavouras do café favorecem o uso de estratégias para controle, no caso de $\boldsymbol{N}$. curtus se tornar praga de cafeeiro nesse Estado.

\section{REFERÊNCIAS}

BACCA, T. et al. Optimum spacing of pheromone traps for monitoring the coffee leaf miner Leucoptera coffeella. Entomologia Experimentalis et Applicata, v.119, n.1, p.3945, 2006. Disponível em: <http://www3.interscience.wiley.com/ cgi-bin/fulltext/118569571/htmlstart>. Acesso em: 08 jun. 2009. doi: 10.1111/j.1570-7458.2006.00389.x.

BUSOLI, A.C. et al. O bicudo do algodoeiro e seu manejo. Jaboticabal : FUNEP, 1994. 32p. (Boletim Técnico, 5).

CAMPANHOLA, C. Compromissos internacionais: convenção sobre diversidade biológica. In: MANZATTO, C.V. et al. Uso agrícola dos solos brasileiros. Rio de Janeiro: Embrapa Solos, 2002. p.135-144.
LANTERI, A.A. et al. Weevils injurious for roots of citrus in São Paulo State, Brazil. Neotropical Entomology, v.31, n.4, p.561-569, 2002. Disponível em: <http://www.scielo.br/ scielo.php ? s cript = sci_art text \& pid = S 1519 $566 \times 2002000400008 \& \operatorname{lng}=\mathrm{en} \& n r m=$ iso\&tlng $=\mathrm{en}>$. Acesso em: 08 jul. 2009. doi: 10.1590/S1519-566X2002000400008.

NAKANO, O. et al. Pragas de solo. Piracicaba: Esalq, 2001. $1 \mathrm{v}$.

OLIVEIRA, D.M.E. et al. Association of the soil bug Atarsocoris sp. (Hemiptera: Cydnidae) with the weed Senecio brasiliensis Less. Neotropical Entomology, v.32, n.1, p.155-157, 2003. Disponível em: <http:/www.scielo.br/scielo.php?script=sci_arttext\&pid=S1519566X2003000100024\&lng=en\&nrm=iso\&tlng=en>. Acesso em: 08 jul. 2009. doi: 10.1590/S1519-566X2003000100024.

SÁNCHEZ-SOTO, S. et al. Ocorrência de Naupactus curtus Boheman (Coleoptera: Curculionidae) em três plantas de importância econômica no Brasil. Neotropical Entomology, v.34, n.4, p.693-693, 2005. Disponível em: <http:// www.scielo.br/scielo.php?script=sci_arttext\&pid=S1519$566 X 2005000400022 \& \operatorname{lng}=e n \& n r m=i s o \& t \operatorname{lng}=p t>$. Acesso em: 08 jul. 2009. doi: 10.1590/S1519-566X2005000400022.

STINNER, R.E. Dispersal and movement of insect pests. Annual Review of Entomology, v.28, n.1, p.319-335, 1983. Disponível em: <http://arjournals.annualreviews.org/doi/pdf/ 10.1146/annurev.en.28.010183.001535>. Acesso em: 08 jul. 2009. doi: 10.1146/annurev.en.28.010183.001535.

WALLNER, W.E. Factors affecting insect population dynamics: differences between outbreak and non-outbreak species. Annual Review of Entomology, n.1, v.32, p.317-40, 1987. Disponível em: <http://arjournals.annualreviews.org/doi/pdf/10.1146/ annurev.en.32.010187.001533>. Acesso em: 08 jul. 2009. doi: 10.1146/annurev.en.32.010187.001533.

WILLIAMS, K. et al. Influence of elevation on bark beetle (Coleoptera: Curculionidae, Scolytinae) community structure and flight periodicity in ponderosa pine forests of Arizona. Environmental Entomology, v.37, n.1, p.94-109, 2008. Disponível em: <http://www.fs.fed.us/rm/pubs_other/ rmrs_2008_williams_k001.pdf $>$. Acesso em: 08 jul. 2009. doi: 10.1603/0046-225X(2008)37[94:IOEOBB]2.0.CO;2. 